\title{
The Traveling Wave Solutions for Some Nonlinear PDEs in Mathematical Physics
}

\author{
Khaled A. Gepreel ${ }^{1,2}$, Saleh Omran ${ }^{1,3}$, Sayed K. Elagan ${ }^{1,4}$ \\ ${ }^{1}$ Mathematics Department, Faculty of Science, Taif University, El-Taif, Kingdom of Saudi Arabia \\ ${ }^{2}$ Mathematics Department, Faculty of Science, Zagazig University, Zagazig, Egypt \\ ${ }^{3}$ Mathematics Department, Faculty of Science, South Valley University, Egypt \\ ${ }^{4}$ Mathematics Department, Faculty of Science,Menofia University, Egypt \\ E-mail: kagepreel@yahoo.com
}

Received December 4, 2010; revised January 17, 2011; accepted January 21, 2011

\begin{abstract}
In the present article, we construct the exact traveling wave solutions of some nonlinear PDEs in the mathematical physics via $(1+1)$ dimensional Kaup Kupershmit equation, the $(1+1)$ dimensional seventh order $\mathrm{KdV}$ equation and $(1+1)$ dimensional Kersten-Krasil Shchik equations by using the modified truncated expansion method. New exact solutions of these equations are found.
\end{abstract}

Keywords: Conjugate Modified Truncated Expansion Method, Traveling Wave Solutions, Nonlinear Evolution Equations

\section{Introduction}

Nonlinear partial differential equations are known to describe a wide variety of phenomena not only in physics -where applications extend over magneto fluid dynamics, water surface gravity waves, electromagnetic radiation reactions, and ion acoustic waves in plasma, just to name a few- but also in biology, chemistry and several other fields. It is one of the important tasks in the study of nonlinear partial differential equations to seek exact and explicit solutions. In the past several decades, both mathematicians and physicists have made many attempts in this direction. Various methods for obtaining exact solutions to nonlinear partial differential equations have been proposed. These are some of them: the truncated expansion method [1-4], the simplest equation method [5], an automated tanh-function method [6], the polygons method [7], and the Clarkson-Kruskal direct method [8]. In this paper, we use a modification of the truncated expansion method introduced in $[9,10]$ to find the exact solutions of the following nonlinear partial differential equations in mathematical physics.

1) The $(1+1)$ dimensional Kaup Kupershmit equation [11]:

$$
-u_{t}+5 u^{2} u_{x}+\frac{25}{2} u_{x} u_{2 x}+5 u u_{3 x}+u_{5 x}=0,
$$

2) The $(1+1)$ dimensional seventh order KdV Equation [12]:

$$
\begin{aligned}
& u_{t}+a u^{3} u_{x}+b u_{x}^{3}+c u u_{x} u_{2 x}+d u^{2} u_{3 x} \\
& +e u_{2 x} u_{3 x}+f u_{x} u_{4 x}+g u u_{5 x}+u_{7 x}=0
\end{aligned}
$$

where $a, b, c, d, e, f$ and $g$ are arbitrary constants.

3) The $(1+1)$ dimensional Kersten-Krasil Shchik equations $[13,14]$ :

$u_{t}+u_{3 x}-6 u u_{x}+3 W W_{3 x}+3 W_{x} W_{2 x}-3 u_{x} W^{2}-6 u W W_{x}=0$, $W_{t}+W_{3 x}-3 W^{2} W_{x}-3 u W_{x}-3 W u_{x}=0$.

The modification of this method allows us to transform this system of differential equations to a system of algebraic equations. As a result we have essential simplification of solutions construction procedure.

\section{The Modification of the Truncated Expansion Method}

Let us present the modification of the truncated expansion method $[9,10]$.

Suppose we have the following nonlinear partial differential equation

$$
F\left(u, u_{t}, u_{x}, u_{x x}, u_{x t}, u_{t t}, \cdots\right)=0,
$$

where $u=u(x, t)$ is an unknown function, $F$ is a polynomial in $u(x, t)$ and its partial derivatives in which the highest order derivatives and the nonlinear terms are involved. 
The traveling wave variable

$$
u(x, t)=Y(z), z=k x-\omega t,
$$

where $k$ and $\omega$ are constants, permits us to reduce Equation (2.1) to an ODE for $u=Y(z)$ in the form

$$
P\left(Y, Y^{\prime}, Y^{\prime \prime}, Y^{\prime \prime \prime}, \cdots\right)=0 \text {, }
$$

where ' $=\frac{d}{d z}$. The modification of the truncated expansion method contains the following steps $[9,10]$.

Step 1. Determination of the dominant term with highest order of singularity. To find the dominant terms we substitute

$$
Y(z)=z^{-p},
$$

into all terms of Equation (2.3). Then we should compare the degrees of all the terms of Equation (2.3) and choose two or more with the lowest degree. The maximum value of $p$ is the pole of Equation (2.3) and we denote it by $N$. It should be noted that this method can be applied when $N$ is an integer. In order to apply this method when $N$ is equal to a fraction or negative integer, we make the following transformation:

1) When $N=\frac{q}{g}$, where $\frac{q}{g}$ is a fraction in lowest term we take the transformation $Y(z)=\phi^{q / g}(z)$.

2) When $N$ is negative integer, we take the transformation $Y(z)=\phi^{N}(z)$. and return to determine the value of $N$ again from the new equation

Step 2. We look for the exact solution of Equation (2.3) in the form

$$
Y(z)=a_{0}+a_{1} Q(z)+a_{2}[Q(z)]^{2}+\cdots+a_{N}[Q(z)]^{N},
$$

where $a_{i}(i=0,1, \cdots, N)$ are arbitrary constants to be determined later and $Q(z)$ equals the following function:

$$
Q(z)=\frac{1}{1+e^{z}}
$$

Step 3. We calculate the necessary number of derivatives of the function $Y(z)$ (using Maple or Mathematica for example). Using the case $N=2$, we get some derivatives of the function $Y(z)$ as follows:

$$
\begin{aligned}
& Y(z)=a_{0}+a_{1} Q(z)+a_{2}[Q(z)]^{2}, \\
& Y^{\prime}(z)=-a_{1} \mathrm{Q}+\left(a_{1}-2 a_{2}\right) \mathrm{Q}^{2}+2 a_{2} \mathrm{Q}^{3}, \\
& Y^{\prime \prime}(z)=a_{1} Q(z)+\left(4 a_{2}-3 a_{1}\right) Q^{2}+\left(2 a_{1}-10 a_{2}\right) Q^{3}+6 a_{2} Q^{4},
\end{aligned}
$$

and so on.

Step 4. We substitute expressions (2.5)-(2.7) to Equation (2.1). Then we collect all terms with the same power in the function $Q(z)$ and equate the expressions to zero.
As a result we obtain an algebraic system of equations. Solving this system we get the values of the unknown parameters.

This algorithm can be easily generalized to polynomial differential equations of any order.

\section{Exact Solution of the $(1+1)$ Dimensional Kaup Kupershmit Equation}

The fifth order Kaup-Kupershmit equation is one of the solitonic equations related to the integrable cases of the Henon-Heiles system. Let us find the exact solutions of the $(1+1)$ dimensional Kaup Kupershmit equation by using the modified truncated expansion method. The traveling wave variable (2.2) permits us to reduce Equation (1.1) to an ODE for $u=Y(z)$ in the form

$$
\begin{aligned}
& \omega Y(z)+\frac{5}{3} k Y^{3}(z)+\frac{15}{4} k^{3}\left[Y^{\prime}(z)\right]^{2} \\
& +5 k^{3} Y(z) Y^{\prime \prime}(z)+k^{5} Y^{(5)}(z)+C_{1}=0,
\end{aligned}
$$

where $C_{1}$ is the integration constant. The pole order of Equation (3.1) is $N=2$. So we look for the solution of Equation (3.1) in the form:

$$
Y(z)=a_{0}+a_{1} Q(z)+a_{2}[Q(z)]^{2},
$$

where $a_{0}, a_{1}$ and $a_{2}$ are arbitrary constants. We substitute Equation(3.2), and the derivative equations (such as Equation(2.6) and Equations (2.7)) into Equation (3.1) and collect all terms with the same power in $[Q(z)]^{i}$, $(i=0,1,2, \cdots)$. Equating each coefficient of the polynomial to zero yields a set of simultaneous algebraic equations omitted here for the sake of brevity. Solving these algebraic equations by either Maple or Mathematica, we get the formulae for the solutions of system (3.1) as follows:

Case 1.

$$
a_{2}=-3 k^{2}, a_{1}=3 k^{2}, a_{0}=-\frac{k^{2}}{4}, \omega=-\frac{k^{5}}{16}, C_{1}=\frac{k^{7}}{96},
$$

where $k$ is an arbitrary constant. In this case the solitrary wave solution takes the following form:

$$
Y_{1}(z)=-\frac{k^{2}}{4}+\frac{3 k^{2}}{1+e^{z}}-\frac{3 k^{2}}{\left(1+e^{z}\right)^{2}},
$$

where $z=k x+\frac{t k^{5}}{16}$.

Case 2.

$a_{2}=-24 k^{2}, a_{1}=24 k^{2}, a_{0}=-2 k^{2}, \omega=-11 k^{5}, C_{1}=-\frac{26 k^{7}}{3}$, 
where $k$ is an arbitrary constant. In this case the solitrary wave solution takes the following form:

$$
Y_{2}(z)=-2 k^{2}+\frac{24 k^{2}}{1+e^{z}}-\frac{24 k^{2}}{\left(1+e^{z}\right)^{2}},
$$

where $z=k x+11 k^{5} t$.

\section{Exact Solution of the $(1+1)$ Dimensional Seventh Order KdV Equation}

The seventh-order KdV Equation (1.2) deals with the structural stability of the KdV equation under a singular perturbation. The traveling wave variable (2.2) permits us to reduce Equation (1.2) to an ODE for $u=Y(z)$ in the form

$$
\begin{aligned}
& -\omega Y^{\prime}+k a Y^{3} Y^{\prime}+b k^{3} Y^{\prime 3}+c k^{3} Y Y^{\prime} Y^{\prime \prime}+d k^{3} Y^{2} Y^{(3)} \\
& \quad+e k^{5} Y^{\prime \prime} Y^{(3)}+f k^{5} Y^{\prime} Y^{(4)}+g k^{5} Y Y^{(5)}+k^{7} Y^{(7)}=0,
\end{aligned}
$$

The pole order of Equation (4.1) is $N=2$. We substitute Equation (3.2), and the derivative equations (such as Equation (2.6) and Equations (2.7)) into Equation (4.1) and collect all terms with the same power $[Q(z)]^{i}$, $(i=0,1,2, \cdots)$. Equating each coefficient of the polynomial to zero yields a set of simultaneous algebraic equations omitted here for the sake of brevity. Solving these algebraic equations by either Maple or Mathematica, we get the formulae for the solutions of system (4.1) as follows:

Case 1.

$$
\begin{gathered}
a_{0}=\frac{2265 k^{2}}{2(-138 g+19 f)}, a_{2}=-\frac{129105 k^{2}}{68(-138 g+19 f),} \\
e=-\frac{19192624}{616835} g+\frac{247159}{97395} f, \\
d=-\frac{2(-138 g+19 f)(-7263 g+5054 f)}{5130225}, \\
\omega=-\frac{1205 k^{7}(-4209 g+2014 f)}{817(-138 g+19 f)}, \\
c=\frac{(-138 g+19 f)(-79853027 g+28321666 f)}{1397131275}, \\
a=\frac{136(-138 g+19 f)^{2}(-846129 g+284582 f)}{9493507013625}, \\
b=-\frac{(-138 g+19 f)(-268357809 g+147586870 f)}{15927296535}, \\
a_{1}=0,
\end{gathered}
$$

where $k, g$ and $f$ are arbitrary constants. In this case the solitary wave solution of Equation (1.2) takes the following form

$$
Y(z)=\frac{2265 k^{2}}{2(-138 g+19 f)}-\frac{129105 k^{2}}{68(-138 g+19 f)\left(1+e^{z}\right)^{2}},
$$

where $z=k x+\frac{1205 k^{7} t(-4209 g+2014 f)}{817(-138 g+19 f)}$.

Case 2.

$$
\begin{aligned}
& a_{2}=-\frac{6895 k^{2}}{2(23 g+6 f)}, a_{1}=\frac{6895 k^{2}}{(23 g+6 f)}, \\
& e=-\frac{364}{985} g-\frac{823}{985} f, \quad \omega=k^{7}, \\
& d=-\frac{2(23 g+6 f)(13679 g+228 f)}{6791575}, \\
& c=\frac{(23 g+6 f)(288179 g+10938 f)}{6791575}, a_{0}=0, \\
& b=-\frac{(23 g+6 f)(93187 g+978 f)}{2716630}, \\
& a=\frac{8(23 g+6 f)^{2}(402 f+19271 g)}{46827909625},
\end{aligned}
$$

where $k, g$ and $f$ are arbitrary constants. In this case the solitary wave solution of Equation (1.2) takes the following form

$$
Y(z)=\frac{6895 k^{2}}{(23 g+6 f)\left(1+e^{z}\right)}-\frac{6895 k^{2}}{2(23 g+6 f)\left(1+e^{z}\right)^{2}},
$$

where $z=k x-k^{7} t$.

Case 3.

$$
\begin{aligned}
& a_{2}=-a_{1}, a_{0}=-\frac{a_{1}}{12}, \\
& e=-\frac{\left(a a_{1}^{2}-12 d k^{2} a_{1}^{2}+48 f k^{4} a_{1}-4 c k^{2} a_{1}^{2}-12096 k^{2}\right)}{48 a_{1} k^{4}}, \\
& \omega=\frac{d k^{3} a_{1}^{2}}{144}-\frac{a k a_{1}^{3}}{1728}+k^{7}, \\
& b=\frac{-4032 k^{6}-a a_{1}^{3}+12 d k^{2} a_{1}^{2}+96 f k^{4} a_{1}}{48 k^{2} a_{1}^{2}}, g=0,
\end{aligned}
$$

where $a_{1}, a, k, d, c$ and $f$ are arbitrary constants. In this case the solitary wave solution of Equation (1.2) takes the following form

$$
Y(z)=\frac{a_{1}\left(-1+10 e^{z}-e^{2 z}\right)}{12\left(1+e^{z}\right)^{2}},
$$

where $z=k x-t\left[\frac{d k^{3} a_{1}^{2}}{144}-\frac{a k a_{1}^{3}}{1728}+k^{7}\right]$. Similarly, we can 
write down the other families of exact solutions of Equation (1.2) which are omitted for convenience.

\section{Exact Solution of the $(1+1)$ Dimensional Kersten-Krasil Shchik Equations}

In this section, we find the exact solutions to the $(1+1)$ dimensional Kersten- Krasil Shchik Equations (1.3) by the modified truncated expansion method. The traveling wave variables $u=Y(z), W=H(z)$ and $z=k x-\omega$ permit us to reduce Equations (1.3) to ODEs for $u=Y(z)$ and $W=H(z)$ in the form

$$
\begin{aligned}
& -\omega Y+k^{3} Y^{\prime \prime}-3 k Y^{2}+3 k^{2} H H^{\prime \prime}-3 k Y H^{2}+C_{1}=0, \\
& -\omega H+k^{3} H^{\prime \prime}-k H^{3}-3 k Y H+C_{2}=0,
\end{aligned}
$$

where $C_{1}$ and $C_{2}$ are the integration constants. To find dominant terms we substitute

$Y(z)=z^{-p_{1}}$ and $H(z)=z^{-p_{2}}$ into all terms of Equation (5.1). Then we should compare degrees of all terms of Equations (5.1) and choose two or more with the lowest degree we have

$$
Y(z)=a_{0}+a_{1} Q(z)+a_{2}[Q(z)]^{2}, H(z)=b_{0}+b_{1} Q(z),
$$

where $a_{0}, b_{0}, a_{1}, b_{1}$ and $a_{2}$ are arbitrary constants. We substitute Equations (5.2) into Equation (5.1) and collect all terms with the same power in

$[Q(z)]^{i},(i=0,1,2, \cdots)$. Equating each coefficient of these polynomials to zero yields a set of simultaneous algebraic equations omitted here for the sake of brevity. Solving these algebraic equations by either Maple or Mathematica, we get the formulae for the solutions of system (5.1) as follows:

Case 1.

$$
\begin{aligned}
& a_{2}=-2 b_{0}^{2}, \quad a_{1}=2 b_{0}^{2}, \quad b_{1}=-2 b_{0}, \\
& k= \pm i b_{0}, \omega=\mp i b_{0}^{3}, C_{1}=C_{2}=a_{0}=0,
\end{aligned}
$$

where $b_{0}$ is an arbitrary constant and $i=\sqrt{-1}$. In this case the solitary wave solution takes the following form:

$$
Y(z)=\frac{2 b_{0}^{2} e^{z}}{\left(1+e^{z}\right)^{2}}, \quad H(z)=b_{0}-\frac{b_{0}}{\left(1+e^{z}\right)},
$$

where $z= \pm i b_{0} x \pm i b_{0}^{3} t$.

Case 2.

$$
\begin{gathered}
a_{2}=-4 b_{0}^{2}, \quad a_{1}=4 b_{0}^{2}, \quad b_{1}=-2 b_{0}, \quad k= \pm 2 i b_{0}, \\
\omega=\mp 2 i b_{0}\left(b_{0}^{2}+3 a_{0}\right), C_{1}= \pm 4 i b_{0}^{3} a_{0}, C_{2}=0,
\end{gathered}
$$

where $a_{0}$ and $b_{0}$ are arbitrary constants. In this case the solitary wave solution takes the following form:

$$
\begin{gathered}
Y(z)=a_{0}+\frac{4 b_{0}^{2}}{1+e^{z}}-\frac{4 b_{0}^{2}}{\left(1+e^{z}\right)^{2}}, \\
H(z)=b_{0}-\frac{2 b_{0}}{1+e^{z}},
\end{gathered}
$$

where $z= \pm 2 i b_{0} x \pm 2 i b_{0}\left(b_{0}^{2}+3 a_{0}\right) t$.

Similarly, we can write down the other families of exact solutions of Equation (1.3) which are omitted for convenience.

\section{Conclusions}

In summary, we may conclude that this method is reliable and straightforward solution method to find the traveling waves of nonlinear partial differential equations. This method allowed us to construct the exact solution for some complicated nonlinear evolution equation than exp-function method. The performance of this method is simple, direct and gives more new exact solutions compared to other method.

\section{References}

[1] J. Weiss, M. Tabor and G. Carnevalle, "The Painleve Property for Partial Differential Equations,” Journal of Mathematical Physics, Vol. 24, No. 3, 1983, pp. 522-526. doi:10.1063/1.525721

[2] N. A. Kudryashov, "Exact Soliton Solutions of the Generalized Evolution Equation of Wave Dynamics," Journal of Applied Mathematics and Mechanics, Vol. 52, No. 3, 1988, pp. 361-365. doi:10.1016/0021-8928(88)90090-1

[3] J. Weiss, “The Panleve Property for Partial Differential Equations. II: Backlund Transformation, Lax Pairs, and the Schwarzian Derivative," Journal of Mathematical Physics, Vol. 24, No. 6, 1983, pp. 1405-1413. doi:10.1063/1.525875

[4] N. A. Kudryashov, "Exact Solutions of the Generalized Kuramoto-Sivashinsky Equation,” Physics Letters A, Vol. 147, No. 5-6, 1990, pp. 287-291. doi:10.1016/0375-9601(90)90449-X

[5] N. A. Kudryashov and N. B. Loguinova, "Extended Simpliest Equation Method for Nonlinear Differential Equations," Applied Mathematics and Computation, Vol. 205, No. 1, 2008, pp. 396-402. doi:10.1016/j.amc.2008.08.019

[6] E. J. Parkes and B. R. Duffy, “An Automated Tanhfunction Method for Finding Solitary Wave Solutions to Nonlinear Evolution Equations," Computer Physics Communications, Vol. 98, No. 3, 1996, pp. 288-300. doi:10.1016/0010-4655(96)00104-X

[7] N. A. Kudryashov and M. V. Demina, "Polygons of Differential Equations for Finding Exact Solutions," Chaos, Solitons \& Fractals, Vol. 33, No. 5, 2007. pp. 1480-1496. doi:10.1016/j.chaos.2006.02.012 
[8] P. A. Clarkson and M. D. Kruskal, "New Similarity Reductions of the Boussinesq Equation," Journal of Mathematical Physics, Vol. 30, No. 10, 1989, pp. 2201-2213. doi:10.1063/1.528613

[9] P. N. Ryabov, "Exact Solutions of the KudryashovSinelshchikov Equation," Applied Mathematics and Computation, Vol. 217, No. 7, 2010, pp. 3585-3590. doi:10.1016/j.amc.2010.09.003

[10] N. A. Kudryashov, "Analytical Theory of Nonlinear Differential Equations,” Institute of Computer Investigations, Moscow, 2004.

[11] M. Musette and C. Verhoeven, "Nonlinear Superposition Formula for the Kaup-Kupershmidt Partial Differential Equation,” Physica D, Vol. 144, No. 1-2, 2000, pp. 211220. doi:10.1016/S0167-2789(00)00081-6
[12] A. H. Salas, "Computing Exact Solutions to a Generalized Lax Seventh-Order Forced KdV Equation,” Applied Mathematics and Computation, Vol. 216, No. 8, 2010, pp. 2333-2338. doi:10.1016/j.amc.2010.03.078

[13] E. M. E. Zayed and K. A. Gepreel, "New Applications of an Improved(G'/G)-Expansion Method to Constract the Exact Solutions of Nonlinear PDEs," International Journal of nonlinear Science and Numerical Simulation, Vol. 11, No. 4, 2010, pp. 273-283.

[14] A. K. Kalkanli, S. Y. Sakovich and T. Yurdusen, "Integrability of Kersten-Krasil'shchik Coupled KdV-mKdV Equations: Singularity Analysis and Lax Pair,” Journal of Mathematical Physics, Vol. 44, No. 4, 2003, pp. 17031708. doi:10.1063/1.1558903 